OSU-HEP-08-03

NSF-KITP-08-51

April 12, 2008

\title{
A New Extensions of MSSM: FMSSM
}

\author{
S. Nandi ${ }^{1}$ and Zurab Tavartkiladze ${ }^{2}$ \\ Department of Physics and Oklahoma Center for High Energy Physics, Oklahoma State \\ University, Stillwater, OK 74078, USA
}

\begin{abstract}
We propose an extension of the MSSM by adding vector like 'matter' fields with masses near the TeV scale. This extension allows us to generate the masses of the bottom quark and tau lepton via radiative corrections such that only up type Higgs doublet couples with quarks and leptons. In addition to providing a natural explanation of the hierarchies between $m_{b, \tau}$ and $m_{t}$, this new extension, which we call FMSSM, allows the heavy sector of the MSSM Higgs bosons to be essentially fermiophobic as well as gaugephobic. Moreover, in this scenario there is no upper bound for the parameter $\tan \beta$. FMSSM can be distinguished from the MSSM, and has peculiar and unorthodox signals at the LHC, especially for the Higgs sector.
\end{abstract}

\footnotetext{
${ }^{1}$ E-mail: s.nandi@okstate.edu

${ }^{2}$ E-mail: zurab.tavartkiladze@okstate.edu
} 


\section{Introduction}

All experimental results to date are in excellent agreement with the predictions of the Standard Model (SM). One essential ingredient of the SM, the existence of the Higgs boson as well as its interactions with the SM particles, are yet to be experimentally established. It is widely believed that the Higgs boson will be discovered at the LHC (even possibly at the Tevatron). However, the most serious theoretical drawback of the SM is the lack of explanation why the Higgs boson's mass is near electro-weak (EW) scale, and not at the Planck scale, the so called hierarchy problem. The supersymmetric (SUSY) extension of the SM is most compelling in the sense that it solves the hierarchy problem naturally. Also the minimal SUSY extension of the SM (MSSM) leads to the successful gauge coupling unification. This gives a compelling ground for grand unification [1]. Moreover, the MSSM provides a viable candidate for the dark matter. In addition, it has a rich spectrum of particles, namely additional Higgs bosons, and the superpartners of the gauge bosons and squarks and sleptons which can be explored at the LHC.

In MSSM the Yukawa couplings are free parameters and should be chosen in such a way as to obtain observed hierarchies between fermion masses and CKM mixing angles. While the top quark mass is close to the EW symmetry breaking scale, the masses of other members of a third family - the bottom quark and tau lepton are smaller by factor of $\sim 60$ and $\sim 140$ respectively. The MSSM parameter $\tan \beta$ can give this mismatch, however, this parameter gets constrained from various observables. This can be avoided, and also $m_{b, \tau} / m_{t} \ll 1$ hierarchies can be explained if $m_{b}$ and $m_{\tau}$ are generated by radiative corrections. Note that, the Yukawa sector of MSSM also does not allow to have the fermiophobic Higgses unlike, for instance, for the general two Higgs doublet model [2]. However, an extension providing radiative bottom-tau mass generation may open such an interesting possibility. Our new extension to the MSSM is motivated by this philosophy.

To achieve all this, we extend the matter sector of MSSM by adding vector-like quarks $D^{c}+\bar{D}^{c}$ and leptons $L+\bar{L}$ with masses at the TeV scale. $D^{c}$ has the same quantum number as the usual $d^{c}$, while $L$ has the same quantum number as the usual leptonic doublet $l$. The top quark has tree level renormalizable coupling with the up type Higgs, however, the bottom quark and the tau lepton has no such tree level coupling with the down type Higgs. Instead they have only tree level coupling with the down type Higgs via $D^{c}$ and $L$ respectively, allowing their mass generation at the one loop level after SUSY breaking. Besides this, this model allows the possibility of having essentially a fermiophobic heavy Higgs sector which is not possible in the MSSM. The phenomenology of this model is very different from the MSSM. In particular, the heavy Higgs signals of fermiophobic MSSM (FMSSM) at the LHC is significantly different from those of MSSM.

\section{Formalism of the model: FMSSM}

We first consider the third generation of quarks and leptons, which in MSSM have the strongest couplings to the Higgs doublets. The quark superfields are $q_{3}, u_{3}^{c}, d_{3}^{c}$ while $l_{3}$ and $e_{3}^{c}$ are the lepton superfields. As in the MSSM, our model has two Higgs doublet superfields, $\hat{h}_{u}$ and $\hat{h}_{d}$. In the MSSM, $\hat{h}_{u}$ couples to the up type quark, while $\hat{h}_{d}$ couples to the down type quarks and charged leptons. In our model, similar to the MSSM, the top quark mass is generated through the renormalizable Yukawa superpotential term

$$
W_{u p}=\lambda_{t} q_{3} u_{3}^{c} \hat{h}_{u} .
$$


In difference from MSSM, in our model, $\hat{h}_{d}$ does not directly couple to the light matter. The masses of the bottom quark and the tau lepton will be generated via radiative corrections. Namely, after SUSY breaking, at the one loop level, the operators $q_{3} d_{3}^{c} h_{u}^{\dagger}$ and $l_{3} e_{3}^{c} h_{u}^{\dagger}$ will be induced, where $h_{u}$ denote the scalar component of the superfield $\hat{h}_{u}$ and remaining states here stand for denoting fermionic components. Since these coupling will be suppressed by loop factors, the natural explanation of the hierarchies $\frac{m_{b}}{m_{t}}, \frac{m_{\tau}}{m_{t}} \ll 1$ is provided. Moreover, what is perhaps most interesting, only $h_{u}$ doublet couples to the fermions. This will allow us to have an essentially fermiophobic as well as gaugephobic heavy Higgs system $\left(H, A\right.$ and $\left.H^{ \pm}\right)$as we will see shortly.

To generate the couplings mentioned above, we extend the fermionic matter sector of the MSSM by adding the vector like matter superfields $D^{c}+\bar{D}^{c}$ and $L+\bar{L}$. Transformation properties of $D^{c}$ and $L$ under $S U(3)_{c} \times S U(2)_{L} \times U(1)_{Y}$ coincide with transformations of $d^{c}$ and $l$ respectively. Thus, these introduced states effectively constitute $S U(5)$ complete multiplets $\overline{5}+5$ [with $\left(D^{c}, L\right) \subset \overline{5}$ and $\left.\left(\bar{D}^{c}, \bar{L}\right) \subset 5\right]$. As we have mentioned, $\hat{h}_{d}$ does not couple directly with light matter, and the states $D^{c}, L$ should do the job for the generation of $m_{b, \tau}$. For this, specific superpotential as well as soft SUSY breaking terms should be introduced. At the same time, absence of any interaction should be justified. For this purpose we will use $R$-symmetry. The $\mu$ and $B_{\mu}$ terms will be generated after SUSY breaking. Since the breaking of $R$-symmetry is an essential ingredient of the SUSY breaking [3], it is advantageous to use $R$-symmetry also for other phenomenological purposes. In our construction we will follow this strategy.

For the usual MSSM fields, and for the new fields that we introduce, we make the following $R$-charge assignment.

$$
\begin{gathered}
R(q)=R\left(u^{c}\right)=R\left(e^{c}\right)=R\left(D^{c}\right)=R\left(\bar{D}^{c}\right)=R(L)=R(\bar{L})=1, \\
R\left(d^{c}\right)=R(l)=r, \quad R\left(\hat{h}_{u}\right)=R\left(\hat{h}_{d}\right)=0,
\end{gathered}
$$

where $r$ is some phase (undetermined for time being). Here we consider family independent $R$ symmetry. We demand that the Lagrangian to be invariant under this $\mathrm{R}$ symmetry. This assignment is compatible with the superpotential term of Eq. (1). In addition, the following superpotential couplings are allowed

$$
W^{\prime}=\lambda_{D} q_{3} D^{c} \hat{h}_{d}+\lambda_{L} L e^{c} \hat{h}_{d}+M_{D} D^{c} \bar{D}^{c}+M_{L} L \bar{L}
$$

As we see, the direct coupling of $h_{d}$ with the light matter is forbidden. Also, the $\mu$ term is not allowed in the superpotential. $\mu$ and $B_{\mu}$ terms, as we have mentioned, will be generated after SUSY breaking by the higher order operators, similar to the proposal of Ref. [4]. Thus we introduce two spurion superfields $X$ and $Y$ with

$$
\langle X\rangle=M^{(X)}+\theta^{2} m M_{\mathrm{Pl}}, \quad\langle Y\rangle=M^{(Y)}+\theta^{2} m M_{\mathrm{Pl}},
$$

and $R$-charges

$$
R(X)=0, \quad R(Y)=r-1 .
$$

As we see, the SUSY breaking is by $F$-terms and the $R$-symmetry is also broken by the VEVs of (4). We assume that a hidden sector is arranged is such a way that the configuration (4) is insured. Note also that there should be included the constant part of the superpotential [5] in order to be able to set the cosmological constant to zero and get the Minkowski vacuum. This 
constant superpotential, breaking the $R$-symmetry explicitly, avoids pseudogoldstones ( $R$-axions), and renders all the phenomenology discussed below intact. In (4) $M_{\mathrm{Pl}} \simeq 2.4 \cdot 10^{18} \mathrm{GeV}$ is the reduced Planck mass, while $m \sim 1 \mathrm{TeV}^{3}$. Through the couplings

$$
\int d^{4} \theta \frac{X^{\dagger}}{M_{\mathrm{Pl}}} \hat{h}_{u} \hat{h}_{d}, \quad \int d^{4} \theta \frac{X^{\dagger} X}{M_{\mathrm{Pl}}^{2}} \hat{h}_{u} \hat{h}_{d}
$$

after substituting the VEVs of Eq. (4), we have $\mu \sim m$ and $B_{\mu} \sim m^{2}$. Furthermore, the operators $\int d^{2} \theta \frac{X}{M_{\mathrm{Pl}}} W_{a} W_{a}$ (where $W_{a}$ is the chiral gauge superfield) generate the gaugino masses $M_{a} \sim m$. The trilinear soft SUSY breaking terms will be generated from the operators $\int d^{2} \theta \frac{X}{M_{\mathrm{Pl}}} W$ where under $W$ we denote all the terms which are included in the superpotential. The mass ${ }^{2}$ soft SUSY breaking terms are induced through the following operators

$$
\begin{gathered}
\int d^{4} \theta \frac{X^{\dagger} X}{M_{\mathrm{Pl}}^{2}} f^{\dagger} f, \quad \text { with } f=\left(q, u^{c}, d^{c}, l, e^{c}, D^{c}, \bar{D}^{c}, L, \bar{L}, \hat{h_{u}}, \hat{h}_{d}\right), \\
\int d^{4} \theta\left(\frac{X^{\dagger} Y}{M_{\mathrm{Pl}}^{2}}\left(D^{c} d_{3}^{c \dagger}+L l_{3}^{\dagger}\right)+\frac{X Y^{\dagger}}{M_{\mathrm{Pl}}^{2}}\left(D^{c \dagger} d_{3}^{c}+L^{\dagger} l_{3}\right)\right) .
\end{gathered}
$$

With these we will have the following terms

$$
\begin{gathered}
m_{\tilde{q}_{3}}^{2}\left|\tilde{q}_{3}\right|^{2}+m_{\tilde{d}_{3}^{c}}^{2}\left|\tilde{d}_{3}^{c}\right|^{2}+m_{\tilde{l}_{3}}^{2}\left|\tilde{l}_{3}\right|^{2}+m_{\tilde{e}_{3}^{c}}^{2}\left|\tilde{e}_{3}^{c}\right|^{2}+ \\
\left(m_{d D}^{2} \tilde{d}_{3}^{c *} \tilde{D}^{c}+m_{l L}^{2} \tilde{l}_{3}^{*} \tilde{L}^{2}+\text { h.c. }\right)
\end{gathered}
$$

with $m_{\tilde{q}_{3}}^{2} \sim m_{\tilde{d}_{3}^{c}}^{2} \sim m_{\tilde{l}_{3}}^{2} \sim m_{\tilde{e}_{3}^{c}}^{2} \sim m_{d D}^{2} \sim m_{l L}^{2} \sim m^{2}$. (We list here only soft mass terms relevant for $m_{b, \tau}$ generation and omit terms like $m_{\tilde{D}^{c}}^{2}\left|\tilde{D}^{c}\right|^{2}, m_{\tilde{L}}^{2}|\tilde{L}|^{2}$, etc.).

It is easy to see that the superpotential term in Eqs. (1), (3) together with the soft breaking terms in Eq. (7), at 1-loop level, generate the operators

$$
\lambda_{b} q_{3} d_{3}^{c} h_{u}^{\dagger}, \quad \lambda_{\tau} l_{3} e_{3}^{c} h_{u}^{\dagger}
$$

with

$$
\begin{gathered}
\lambda_{b}=\frac{\lambda_{D} \alpha_{3}}{4 \pi} \frac{8}{3} \mu \kappa M_{\tilde{g}} \frac{m_{d D}^{2}}{m_{\tilde{q}_{3}}^{4}} I_{q}, \quad \lambda_{\tau}=\frac{\lambda_{L} \alpha_{1}}{4 \pi} \frac{3}{5} \mu \kappa M_{\tilde{B}} \frac{m_{l L}^{2}}{m_{\tilde{e}_{3}^{c}}^{4}} I_{l} \\
I_{q}=I\left(\frac{M_{\tilde{g}}^{2}}{m_{\tilde{q}_{3}}^{2}}, \frac{M_{D}^{2}+m_{\tilde{D}^{c}}^{2}}{m_{\tilde{q}_{3}}^{2}}, \frac{m_{\tilde{d}_{3}^{c}}^{2}}{m_{\tilde{q}_{3}}^{2}}, \frac{m_{d D}^{4}}{m_{\tilde{q_{3}}}^{4}}\right) \quad I_{l}=I\left(\frac{M_{\tilde{\tilde{q}}}^{2}}{m_{\tilde{e}_{3}^{c}}^{2}}, \frac{M_{L}^{2}+m_{\tilde{L}}^{2}}{m_{\tilde{e}_{3}^{c}}^{2}}, \frac{m_{\tilde{l}_{3}}^{2}}{m_{\tilde{e}_{3}^{c}}^{2}}, \frac{m_{l L}^{4}}{m_{\tilde{e}_{3}^{c}}^{4}}\right), \\
I(a, b, c, d)=\int_{0}^{\infty} \frac{t d t}{t+1} \frac{1}{t+a} \frac{1}{(t+b)(t+c)-d} .
\end{gathered}
$$

The corresponding diagrams are shown in Fig. 1. The vortex involving $h_{u}^{\dagger}$ emerges from the potential term $\left|F_{h_{d}}\right|^{2}=\left|\lambda_{D} \tilde{q}_{3} \tilde{D}^{c}+\lambda_{L} \tilde{L} \tilde{e}_{3}^{c}+\kappa \mu h_{u}\right|^{2} \rightarrow \mu \kappa\left(\lambda_{D} \tilde{q}_{3} \tilde{D}^{c} h_{u}^{\dagger}+\lambda_{L} \tilde{L} \tilde{e}_{3}^{c} h_{u}^{\dagger}\right)$, where $\kappa$ is some dimensionless constant of the order of one. The operator, $\lambda^{(1)} q_{3} d_{3}^{c} h_{d}$ (with $\lambda^{(1)} \sim \lambda_{b} \frac{\langle Y\rangle}{M_{\mathrm{Pl}}}$ ) generated

\footnotetext{
${ }^{3}$ Here, for simplicity, we assume gravity mediated SUSY breaking scenario. However, in this framework different SUSY breaking scenarios can work as well.
} 


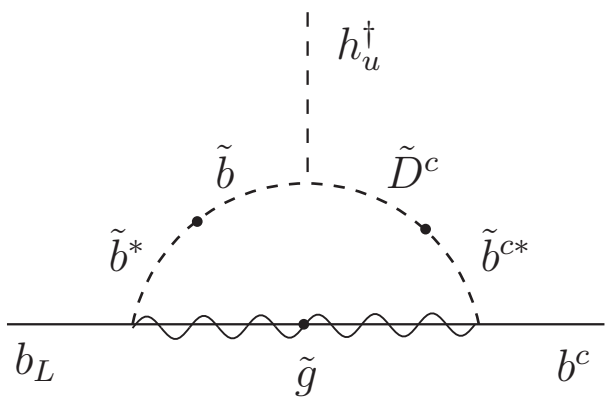

(a)

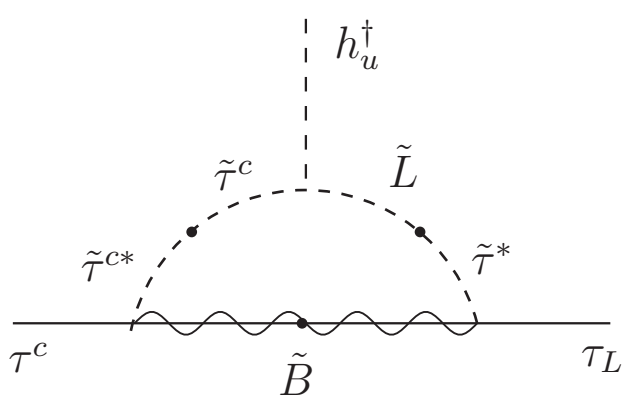

(b)

Figure 1: Diagrams generating bottom quark and tau lepton masses.

by the loops, will be suppressed for small values of $\frac{\langle Y\rangle}{M_{\mathrm{P} 1}}=\frac{M^{(Y)}}{M_{\mathrm{Pl}}}$. To be more specific, this coupling induces the correction to the bottom quark mass $\delta m_{b} \sim m_{b} \frac{\langle Y\rangle}{M_{\mathrm{Pl}}} \frac{1}{\tan \beta}$. Already for $\tan \beta \sim 10$, this correction will be negligible with $\frac{\langle Y\rangle}{M_{\mathrm{P} 1}} \lesssim 0.1$. Moreover, the $\lambda^{(1)}$ coupling would induce corrections to the matter-Higgs interactions. For instance, the $b \bar{b}$ interaction with heavy $\mathrm{CP}$ even neutral Higgs $(H)$ will receive the correction $\delta \lambda_{b \bar{b} H} \sim \frac{m_{b}}{\sqrt{2} v} \frac{\langle Y\rangle}{M_{\mathrm{Pl}}}$, which still will not give anything new if $\frac{\langle Y\rangle}{M_{\mathrm{P} 1}} \lesssim \frac{m_{h}^{2}}{m_{H}^{2}} \lesssim \frac{1}{50}$. The latter ratio controlls the suppression factor of the heavy Higgs fields' interaction with the matter (see discussion below). The value of $\langle Y\rangle \lesssim M_{\mathrm{Pl}} / 50$ is not unnatural. Since the VEV $\langle Y\rangle$ should depend on the specific superpotential couplings, with the dimensionless couplings of the order of $1 / 3-3$ and mass terms with values $M_{\mathrm{Pl}} / 5$ say, the suppression factor $1 / 50$ can be easily emerged without any fine tunings. Thus, with such suppressed $\langle Y\rangle\left(\lesssim M_{\mathrm{Pl}} / 50\right)$, the operator $\lambda^{(1)} q_{3} d_{3}^{c} h_{d}$ becomes phenomenologically unimportant.

Note that if the additional vector-like particle $\left(D^{c}\right.$ and $\left.\mathrm{L}\right)$ masses are in the TeV scale, which we take to be the SUSY breaking scale, we obtain the values of the masses of the bottom quark and the tau lepton in their observed range (with appropriate choices for the couplings $\lambda_{D}, \lambda_{L} \sim 1$ and soft masses). The mixed soft terms $m_{d D}^{2}, m_{l L}^{2}$ in (7) are important for generating these operators. Since these off diagonal conversions involve only third generation and the heavy vector like states, they will not contribute to the FCNC. Thus with our explanation of why the bottom quark and tau are so much lighter compared to the top quark, we expect a vector-like quarks and leptons at the TeV scale. The vector-like quark, being a color triplet, can be copiously pair produced at the LHC. Thus our proposed model can be easily tested. We will discuss the phenomenological implications of our model in section 3.

Now we discuss the coupling of the mass eigenstate Higgs bosons with the 3rd family fermions in our model. We have three neutral physical Higgs bosons $(h, H, A) \equiv \phi$ where $h$ is lightest $\mathrm{CP}$ even Higgs, $H$ is heavy $(\sim 1 \mathrm{TeV}) \mathrm{CP}$ even Higgs while $A$ is $\mathrm{CP}$ odd. From this and the Yukawa couplings given in (1) and (8), we can determine their interaction with third family fermions:

$$
\begin{gathered}
\mathcal{L}_{f_{3} f_{3} \phi}=\frac{m_{t}}{\sqrt{2} v s_{\beta}}\left[\bar{t} t\left(c_{\alpha} h+s_{\alpha} H\right)+\bar{t} i \gamma_{5} t c_{\beta} A\right]+\frac{m_{b}}{\sqrt{2} v s_{\beta}}\left[\bar{b} b\left(c_{\alpha} h+s_{\alpha} H\right)+\bar{b} i \gamma_{5} b c_{\beta} A\right] \\
+\frac{m_{\tau}}{\sqrt{2} v s_{\beta}}\left[\bar{\tau} \tau\left(c_{\alpha} h+s_{\alpha} H\right)+\bar{\tau} i \gamma_{5} \tau c_{\beta} A\right] .
\end{gathered}
$$


The angle $\alpha$ is the mixing angle between the two neutral CP-even Higgs bosons, $\mathrm{h}$ and $\mathrm{H}$.

The interaction of the physical charged Higgs $H^{ \pm}$with the 3rd family fermions is given by

$$
\mathcal{L}_{f_{3} f_{3} H^{ \pm}}=\frac{m_{t}}{v \tan \beta} b_{L} t^{c} H^{+}+\frac{m_{b}}{v \tan \beta}\left(t_{L} b^{c}+\frac{m_{\tau}}{m_{b}} \nu_{\tau} \tau^{c}\right) H^{-}+\text {h.c } .
$$

Here $v \simeq 174 \mathrm{GeV}$ and the angles $\alpha, \beta$ are related as follows

$$
\frac{\sin 2 \alpha}{\sin 2 \beta}=-\frac{m_{H}^{2}+m_{h}^{2}}{m_{H}^{2}-m_{h}^{2}}, \quad \frac{\tan 2 \alpha}{\tan 2 \beta}=\frac{m_{A}^{2}+m_{Z}^{2}}{m_{A}^{2}-m_{Z}^{2}}, \quad 0<\beta<\frac{\pi}{2}, \quad-\frac{\pi}{2}<\alpha<0 .
$$

For $m_{A}, m_{H} \gg m_{Z}, m_{h}$, the relation between the angles $\alpha$ and $\beta$ becomes

$$
\alpha=-\frac{\pi}{2}+\beta+\mathcal{O}\left(\frac{m_{h}^{2}}{m_{H}^{2}}, \frac{m_{Z}^{2}}{m_{A}^{2}}\right) .
$$

Note that, in this limit, the coupling of the light neutral Higgs, $h$, to the EW gauge bosons, which is proportional to $\sin (\beta-\alpha)$ becomes close to the SM values, while the coupling of the heavy Higgs, H, which is proportional to $\cos (\beta-\alpha)$, is $\operatorname{suppressed}\left(\sim \frac{m_{h}^{2}}{m_{H}^{2}}, \frac{m_{Z}^{2}}{m_{A}^{2}}\right)$. In this limit, the Yukawa couplings of the lightest neutral CP even Higgs $(h)$ with the 3rd family of matter fermions become:

$$
\lambda_{t \bar{t} h} \simeq \frac{m_{t}}{\sqrt{2} v}, \quad \lambda_{b \bar{b} h} \simeq \frac{m_{b}}{\sqrt{2} v}, \quad \lambda_{\tau \bar{\tau} h}=\frac{m_{\tau}}{\sqrt{2} v} .
$$

Thus the Yukawa couplings of the light Higgs becomes identical to those in the SM. The coupling of the bottom quarks and the tau lepton is different from MSSM. These are not proportional to $\sqrt{1+\tan ^{2} \beta}$ as in the MSSM. This is because, in our model, the bottom quark and the tau lepton does not couple to $h_{d}$ directly. This is one distinctive feature of our model compared to the MSSM.

The couplings of the heavy Higgs sector to the fermions are given by

$$
\begin{gathered}
\lambda_{t \bar{t} H} \simeq \lambda_{t \bar{t} A}=\frac{\lambda_{b \bar{t} H^{+}}}{\sqrt{2}}=\frac{m_{t}}{\sqrt{2} v} \frac{1}{\tan \beta}, \quad \lambda_{b \bar{b} H} \simeq \lambda_{b \bar{b} A}=\frac{\lambda_{t \bar{b} H^{-}}}{\sqrt{2}}=\frac{m_{b}}{\sqrt{2} v} \frac{1}{\tan \beta}, \\
\lambda_{\tau \bar{\tau} H} \simeq \lambda_{\tau \bar{\tau} A}=\frac{\lambda_{\nu_{\tau} \bar{\tau} H^{-}}}{\sqrt{2}}=\frac{m_{\tau}}{\sqrt{2} v} \frac{1}{\tan \beta} .
\end{gathered}
$$

Note that the superpotential couplings $q_{3} d_{3}^{c} \hat{h}_{d}$ and $l_{3} e_{3}^{c} \hat{h}_{d}$ are forbidden thanks to the $R$ symmetry. By the same token soft SUSY breaking terms $\tilde{q}_{3} \tilde{D}^{c} h_{d}$ and $\tilde{e}_{3}^{c} \tilde{L} h_{d}$ are absent at tree level. With $\tan \beta \rightarrow \infty$, which means that $\left\langle h_{u}^{0}\right\rangle=174 \mathrm{GeV}$ and $\left\langle h_{d}^{0}\right\rangle \rightarrow 0$, all couplings in (15) vanish and $H, A$ and $H^{ \pm}$decouple from the third generation. Thus, in this limit, they become fermiophobic or quasi-fermiophobic. The latter case can be realized if light generations will couple to $h_{d}$. This will depend on how we construct the Yukawa sector for the light families. However, in any case those interactions with Higgses would be strongly suppressed by tiny Yukawa couplings. As far as the very large values of the $\tan \beta$ (or equivalently $\left\langle h_{d}^{0}\right\rangle \ll\left\langle h_{u}^{0}\right\rangle$ ) is concerned this regime is quite realizable. The VEV $\left\langle h_{d}^{0}\right\rangle$ depends on the values of soft mass squires $m_{h_{d}}^{2}, m_{h_{u}}^{2}$ and also on the $B$-term. By proper selection of these parameters the regime $\tan \beta \gg 1$ can be realized. As mentioned earlier, gauge interactions of the heavy Higgses with $W W$ and $Z Z$ are also suppressed 
in this limit. Thus, their productions will be highly suppressed via gluon-gluon fusion or the intermediate vector boson fusion, which are the two dominant mechanism for producing these Higgs bosons.

We will discuss the phenomenological implications of these for the detection of the Higgs bosons at LHC in section 3.

We now discuss the couplings of the Higgs bosons with the new vector-like fermions, $D^{c}$ and $L$. Note that $\hat{h}_{d}$ couples with third generation in combination with heavy states $D^{c}$ and $L$ (see Eq. (3)). From these couplings it is straightforward to derive the Higgs interactions involving one heavy fermion. They are given as follows

$$
\begin{gathered}
\mathcal{L}_{f_{3} F \phi}=\frac{\lambda_{D}}{\sqrt{2}}\left(b_{L} D^{c}+\frac{\lambda_{L}}{\lambda_{D}} \tau^{c} E^{(L)}\right)\left(-s_{\alpha} h+c_{\alpha} H-i s_{\beta} A\right)+\text { h.c. } \\
\mathcal{L}_{f_{3} F H^{ \pm}}=\lambda_{D} s_{\beta} \cdot t_{L} D^{c} H^{-}+\lambda_{L} s_{\beta} \cdot \tau^{c} N^{(L)} H^{-}+\text {h.c. }
\end{gathered}
$$

where $E^{(L)}$ and $N^{(L)}$ denote the charged and neutral components of $L$ (e.g. $L=\left(N^{(L)}, E^{(L)}\right)$ ). Note that in the limit, $m_{A}, m_{H}, m_{H}^{+} \gg m_{Z}, m_{h}$, the coupling of $h$ with $D^{c}$ and b vanishes, while the coupling of $H$ and $A$ become $\approx s_{\beta} \lambda_{D, L} / \sqrt{2}$. Thus, if $D^{c}$ and $L$ states are produced at the LHC, and they are heavier than $H$, they will decay to the heavy Higgs and the bottom quark. On the other hand, if $D^{c}$ is lighter than $H$, then it will decay to the lighter Higgs $h$ and $b$ with a coupling $\approx c_{\beta} \lambda_{D} / \sqrt{2}$ (which gets suppressed for large values of $\tan \beta$ ).

Before closing this section, we give the matter-smatter interaction with Higgsinos. From the superpotential coupling (1), we have the following up type higgsino $\left(\widetilde{h}_{u}\right)$ Yukawa coupling with the quark and squark:

$$
\frac{m_{t}}{v s_{\beta}}\left(\tilde{q}_{3} t^{c}+q_{3} \tilde{t}^{c}\right) \tilde{h}_{u}+\text { h.c. }
$$

Since there is no superpotential coupling of $\hat{h}_{d}$ with light matter, the combinations like $\tilde{q}_{3} b^{c}, \tilde{l}_{3} \tau^{c}$ etc. do not couple with $\widetilde{h}_{d}$. They receive couplings with $\widetilde{h}_{u}$ through 1-loop diagrams (similar to ones given in Fig. 1):

$$
\begin{gathered}
\lambda_{\tilde{q}_{3} b \widetilde{h}_{u}} \tilde{q}_{3}^{*} \bar{b}^{c} \widetilde{h}_{u}+\lambda_{\tilde{\tau}_{3} \widetilde{h}_{u}} \tilde{\tau}^{c *} \bar{l}_{3} \widetilde{h}_{u}+\text { h.c. } \\
\text { with } \quad \lambda_{\tilde{q}_{3} \sigma \widetilde{h}_{u}} \simeq \frac{m_{\tau}}{v s_{\beta}} \frac{\lambda_{D}}{3 \lambda_{L}} \frac{I^{\prime}}{I_{l}}, \quad \lambda_{\tilde{\tau}_{3} \widetilde{h}_{u}} \simeq \frac{m_{\tau}}{v s_{\beta}} \frac{1}{2}\left(\frac{I^{\prime \prime}}{I_{l}}+5 \frac{M_{\tilde{W}}}{M_{\tilde{B}}} \frac{I^{\prime \prime \prime}}{I_{l}}\right),
\end{gathered}
$$

where $I^{\prime}, I^{\prime \prime}, I^{\prime \prime \prime}$ are loop integrals defined similar to those given in Eq. (9).

At one loop level, the couplings of $\tilde{q}_{3} t^{c}, q_{3} \tilde{t}^{c}$ with down type Higgsino $\widetilde{h}_{d}$ are induced:

$$
\begin{aligned}
\lambda_{\tilde{q}_{3} t \widetilde{h}_{d}} \tilde{q}_{3}^{*} \bar{t}^{c} \widetilde{h}_{d}+\lambda_{q_{3} \tilde{t} \tilde{h}_{d}} \bar{q}_{3} \tilde{t}^{c} \widetilde{h}_{d}+\text { h.c. } \\
\text { with } \quad \lambda_{\tilde{q}_{3} t \widetilde{h}_{d}} \sim \lambda_{q_{3} \tilde{\tilde{h}_{d}}} \sim \frac{1}{16 \pi^{2}} \frac{m_{t}}{v s_{\beta}} .
\end{aligned}
$$

Note once more that $\widetilde{h}_{d}$ has no Yukawa interactions with $\tilde{q}_{3} b^{c}, q_{3} \tilde{b}^{c}, \tilde{l}_{3} \tau^{c}$ and $l_{3} \tilde{\tau}^{c}$. Moreover, existing higgsino couplings in (17)-(19) are rather insensitive to the values of $\tan \beta(\gtrsim 2)$. This fact will allow to have no upper bound on $\tan \beta$ and relaxes constrains on some SUSY parameters. We will discuss the phenomenological implications of this in more detail in the next section. 


\section{Phenomenological Implications}

We now briefly discuss some of the phenomenological implications of the model. The model has following major phenomenological implications.

\section{(i) New vector-like quarks and leptons in the $\mathrm{TeV}$ scale}

Within our scenario the bottom quark and the tau lepton masses are generated at 1-loop level. This gives natural way of explaining their suppressed values in comparison of $m_{t}$. In order the mechanism to work, we need new vector like states near $\mathrm{TeV}$ scale. Therefore, we expect to see a vector-like quarks $D^{c}+\bar{D}^{c}$, and a vector-like leptons $L+\bar{L}$ at the TeV scale. The $D^{c}$ under $S U(3)_{C} \times S U(2)_{L} \times U(1)_{Y}$ transforms as $\left(\overline{3}, 1,-\frac{2}{\sqrt{60}}\right)$, where $U(1)_{Y}$ hypercharge is taken with $S U(5)$ normalization. The transformation properties of $L$ state is $\left(1,2, \frac{3}{\sqrt{60}}\right)$. Since $D^{c}$ has strong interactions, it can be pair produced at the LHC up to a mass of about $2 \mathrm{TeV}$. If $D^{c}$ is heavier than $H$, then it will decay dominantly to a b quark and $H$ (see Eq. (16)). It can also decay to the t-quark and the charged Higgs, although this decay mode will be somewhat suppressed compared to $D^{c} \rightarrow b H$. So the dominant final state signal from the $D^{c} D^{c \star}$ pair productions will depend on the decay mode of $H$ ( $D^{c \star}$ should not be confused with $\bar{D}^{c}$, the latter is a 'mirror' of $D^{c}$ ). The coupling for the $H \rightarrow t \bar{t}$ decay is $\left(\frac{m_{t}}{\sqrt{2} v \tan \beta}+\delta_{t \bar{t} H}\right.$ ) (where $\delta_{t \bar{t} H} \sim 10^{-2}$ is coming by the 1-loop induced operator $q_{3} u_{3}^{c} h_{d}^{\dagger}[6,7]$, in analogy of the diagram of Fig. 1a), whereas the coupling for $H \rightarrow W W$ is $\frac{g}{2} \frac{m_{h}{ }^{2}}{M_{H}^{2}}$. Thus the final state signals from the $D^{c} D^{c \star}$ pair productions will be $b \bar{b} t \bar{t}$ or

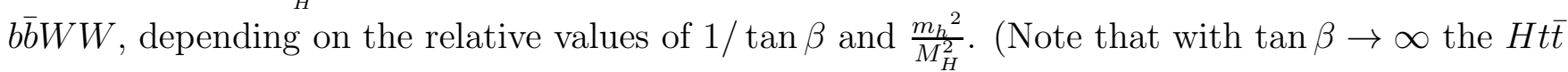
coupling saturates to $\delta_{t \bar{t} H} \sim 10^{-2}$ and can be comparable with $H W W$ coupling). The cross section for the $D^{c} D^{c \star}$ production at the LHC (with $\sqrt{s}=14 \mathrm{TeV}$ ) is about $100 \mathrm{fb}$ [8]. So this could be an observable signal even at the early runs of the LHC with luminosity of few $\mathrm{fb}^{-1}$.

\section{(ii) Different Higgs signals compared to what we expect in the MSSM}

In our model, in the limit of large $\tan \beta$ and $m_{H, A} \gg m_{Z, h}$, the gauge coupling as well as the fermionic coupling of the light Higgs $\mathrm{h}$ is essentially like the SM Higgs. One major difference with MSSM is that the h coupling to $b \bar{b}$ and $\tau \bar{\tau}$ is not $\tan \beta$ enhanced as in the MSSM, since $h_{d}$ does not couple directly to the down type quarks and to the charged leptons in our model. Thus there is no restriction on $\tan \beta$ in our model, either from the perturbativity or unitarity of the $h b \bar{b}$ coupling. Neither the enhancement of the rare processes like $b \rightarrow s \gamma[7,9]$ and $B_{0} \rightarrow \mu^{+} \mu^{-}[10]$ occurs by increase of $\tan \beta$.

In the SM or in MSSM, the pair production for the Higgs bosons have very small cross section, and most likely, will not be observable at the LHC. In our model, because a pair of the Higgs bosons can be produced from the decays of the $D^{c} D^{c \star}$ pairs, and this $D^{c} D^{c \star}$ are produced via strong interaction, the double Higgs production has a sizable cross section at the LHC. For example, for the $D^{c}$ mass of $1 \mathrm{TeV}$, this cross section is about $100 \mathrm{fb}$, while for a $800 \mathrm{GeV} D^{c}$ mass, the cross section is $400 \mathrm{fb}$. This double Higgs production will be the heavy Higgs pair if the $D^{c}$ is heavier than $H$. However, if $H$ is heavier than $D^{c}$, then this double Higgs production [11] will be the light Higgs pair, with anomalously large cross section compared to that expected in the SM, or in the MSSM.

(iii) SUSY signals 
Our model will have different signals also with respect to the detection or/and the production of SUSY particles. For example, as was noted in previous section, the higgsino-fermion-sfermion interactions are practically insensitive to the value of $\tan \beta$. Therefore, any process which involve the neutralino or chargino is not enhanced even for very large values of $\tan \beta$.

For example, after gluino pair production at LHC, each gluino decay in lightest squark $\left(\tilde{q}_{l}\right)$ and corresponding quark: $\tilde{g} \rightarrow \tilde{q}_{l}^{*} q$ provided that $M_{\tilde{g}}>m_{\tilde{q}_{l}}$. With $\tilde{q}_{l}=\tilde{b}^{c}$ and the lightest SUSY particle (LSP) being the neutralino $\left(\widetilde{\chi}_{1}^{0}\right)$, the subsequent decay of $\tilde{b}^{c}$ is through the channel $\tilde{b}^{c} \rightarrow b^{c} \widetilde{\chi}_{1}^{0}$. This decay is only due to gauge coupling $g_{1}$ and completely independent of Yukawa interactions [see (17)-(19)]. Thus, in this case, the process $\tilde{g} \tilde{g} \rightarrow b \bar{b}+\not \mathbb{E}$ is insensitive to the value of $\tan \beta$.

Moreover, for example, if the LSP is $\widetilde{\chi}_{1}^{0}$, then the rare decay of the next heavier neutralino $\widetilde{\chi}_{2}^{0} \rightarrow \widetilde{\chi}_{1}^{0} \gamma$ is not sensitive to $\tan \beta$ unlike the MSSM, in which some restrictions on SUSY parameters should be imposed [12]. Thus many of the constraints in MSSM do not apply in our model.

\section{(iv) Relaxing restrictions from neutralino cold dark matter}

Assuming that LSP is the $\widetilde{\chi}_{1}^{0}$ neutralino, the relic density $\Omega_{\chi} h^{2}$ depends on neutralino couplings. For instance, in MSSM, the $\tilde{\tau}$ being the NLSP, the co-annihilation processes are sensitive to the value of $\tan \beta$. These processes, in different scenarios, constrain the value of $\tan \beta$ and different SUSY parameters [13], [12]. In our scenario, as we have seen, the higgsono couplings are rather insensitive to $\tan \beta$ (see Eqs. (17)-(19)) and therefore no such constraints would apply. The detailed investigation of a relic density for neutralino (or other candidate) cold dark matter within proposed scenario is beyond the scope of this work.

\section{Inclusion of Light Families}

Now let us discuss the possible mass generation for the light families. This must be done in such a way as not to cause any phenomenological inconsistency. Here we present one possible way which leads to realistic phenomenology. We assume that all three families of the down type quarks and charged leptons obtain masses radiatively (similar to the bottom quark and tau lepton) by introducing additional vector like states near $\mathrm{TeV}$ scale. Thus, we introduce three vector like pairs $\left(D^{c}+\bar{D}^{c}\right)_{i},(L+\bar{L})_{i}$ with $i=1,2,3$. Without any selection rule, these new states may be a new source for flavor violation. To avoid this some care should be exercised. Thus we postulate a flavor symmetry $S O(3)$ which will guarantee the flavor conservation. Three families of quarks and leptons and additional vector like pair are triplets 3 of $S O(3)$ while the higgs superfields are the singlets. Thus, all bi-linear (mass or mass ${ }^{2}$ ) couplings are universal and diagonal. For desirable fermion masses and mixings, we introduce the flavon superfields $\xi$ and $\chi$ in the fundamental 3-plet and symmetric 5-plet representations of $S O(3)$ respectively. The superpotential couplings relevant for the up type quark sector are

$$
\frac{1}{M}\left(\alpha_{M} M \mathbf{1}+\alpha_{u} \xi+\beta_{u} \chi\right)_{i j} q_{i} u_{j}^{c} \hat{h}_{u}
$$

where $M$ is some cut off scale and $\alpha_{M}, \alpha_{u}, \beta_{u}$ some dimensionless couplings. Clearly, for obtaining the observed values of the masses $m_{u, c, t}$, proper selection of the $\operatorname{VEVs}\langle\xi\rangle,\langle\chi\rangle$ and the couplings

$\alpha_{M, u}, \beta_{u}$ is needed. For this purpose, details of $S O(3)$ flavor symmetry breaking need to be addressed (in a spirit of Ref. [15]), which is beyond the scope of this work. Now let us turn to the down quark 
sector. The relevant superpotential couplings are

$$
\frac{\lambda_{D}}{M^{\prime}}\left(M^{\prime} \mathbf{1}+\alpha_{d} \xi+\beta_{d} \chi\right)_{i j} q_{i} D_{j}^{c} \hat{h}_{d}+M_{D} D_{i}^{c} \bar{D}_{i}^{c},
$$

while the relevant soft SUSY breaking terms are

$$
m_{\tilde{q}}^{2}\left|\tilde{q}_{i}\right|^{2}+m_{\tilde{d}^{c}}^{2}\left|\tilde{d}_{i}^{c}\right|^{2}+m_{d D}^{2}\left(\tilde{d}_{i}^{* c} \tilde{D}_{i}^{c}+\tilde{d}_{i}^{c} \tilde{D}_{i}^{* c}\right)+m_{\tilde{D}^{c}}^{2}\left|\tilde{D}_{i}^{c}\right|^{2}+m_{\tilde{D}^{c}}^{2}\left|\tilde{\bar{D}}_{i}^{c}\right|^{2} .
$$

By the loops similar to one given in Fig. 1a the operator $\propto \frac{\lambda_{D}}{M^{\prime}}\left(M^{\prime} \mathbf{1}+\alpha_{d} \xi+\beta_{d} \chi\right)_{i j} q_{i} d_{j}^{c} h_{u}^{\dagger}$ will be generated which will give masses to all light down type quarks. Note the crucial point, that all mass couplings in (21) and (22) are degenerate. Therefore, after diagonalization of the quark mass matrices, all these bilinear couplings will remain diagonal and will not cause any additional flavor violation. This is indeed the merit of the $S O(3)$ flavor symmetry in our construction ${ }^{4}$.

The charged lepton sector can be constructed in a same manner. The relevant superpotential and soft SUSY breaking operators are respectively

$$
\begin{gathered}
\frac{\lambda_{L}}{M^{\prime \prime}}\left(M^{\prime \prime} 1+\alpha_{l} \xi+\beta_{l} \chi\right)_{i j} L_{i} e_{j}^{c} \hat{h}_{d}+M_{L} L_{i} \bar{L}_{i}, \\
m_{\tilde{l}}^{2}\left|\tilde{l}_{i}\right|^{2}+m_{\tilde{e}^{c}}^{2}\left|\tilde{e}_{i}^{c}\right|^{2}+m_{l L}^{2}\left(\tilde{l}_{i}^{*} \tilde{L}_{i}+\tilde{l}_{i} \tilde{L}_{i}^{*}\right)+m_{\tilde{L}}^{2}\left|\tilde{L}_{i}\right|^{2}+m_{\tilde{L}^{2}}^{2}\left|\tilde{\bar{L}}_{i}\right|^{2} .
\end{gathered}
$$

One can check that the operator $\propto \frac{\lambda_{L}}{M^{\prime \prime}}\left(M^{\prime \prime} 1+\alpha_{l} \xi+\beta_{l} \chi\right)_{i j} l_{i} e_{j}^{c} h_{u}^{\dagger}$ will be generated by the loops similar to one given in Fig. 1b. The flavor is still conserved due to mass operator degeneracies in (23) and (24) insured by $S O(3)$ flavor symmetry.

Finally, let us point out that the $R$-symmetry applied and discussed in sect. 2 works out also for three families and is compatible with $S O(3)$ flavor symmetry.

\section{$5 \quad$ Embedding in $S U(5)$ GUT}

Grand unified theories have many virtues [1]. It is highly motivated to extend phenomenologically interesting scenario to the GUT. As it turns out, it is straightforward to embed the scenario discussed in this paper in the grand unification. Let us demonstrate this as an example for SUSY $S U(5)$. As we know, the matter sector of minimal $S U(5)$ consists of one $(10+\overline{5})$ per generation: $10_{i}=\left(q, u^{c}, e^{c}\right)_{i}$ and $\overline{5}_{i}=\left(d^{c}, l\right)_{i}(i=1,2,3)$. Since in the previous section we have seen that inclusion of all three families of quarks and leptons is possible, here we make presentation only with a single family. The up and down Higgs supermultiplets $h_{u}$ and $h_{d}$ are embedded in $H(5)$ and $\bar{H}(\overline{5})$ respectively. We extend the matter sector with the vector like pair $\bar{F}(\overline{5})+F(5)$ with a composition: $\bar{F}=\left(D^{c}, L\right), F=\left(\bar{D}^{c}, \bar{L}\right)$. The up type quark mass is generated through the superpotential coupling

$$
10 \cdot 10 \mathrm{H} \text {. }
$$

This operator includes the interaction of Eq. (1). In order to realize the mechanism proposed in Section 2, one should not couple $\bar{H}$ directly to the light matter (all this still can be suitably

\footnotetext{
${ }^{4}$ In various constructions, the same flavor symmetry has been proven to be very useful for solving SUSY flavor problem [14] and also building predictive fermion mass pattern [15].
} 
achieved by $R$-symmetry). Therefore, down quark and charged lepton masses should be generated radiatively. Thus we introduce the following $S U(5)$ invariant superpotential couplings

$$
\lambda_{F} F 10 \bar{H}+M_{F} \bar{F} F
$$

and the following soft SUSY breaking terms

$$
m_{10}^{2}|\tilde{10}|^{2}+m_{\overline{5}}|\tilde{\overline{5}}|^{2}+m_{5 F}^{2}\left(\tilde{\widetilde{5}} \tilde{\bar{F}}^{*}+\tilde{\overline{5}}^{*} \tilde{\bar{F}}\right) .
$$

We assume that the mass $M_{F}$ is close to $\mathrm{TeV}$ scale and also the $\mu$ term has the same magnitude. The latter will be the case by achieving the doublet-triplet splitting (for instance through the superpotential couplings $\bar{H}\left(M_{H}+24_{H}\right) H$ by fine tuning). With these, we can see that all terms of (1)-(7) can be reproduced and the radiative generation of $m_{b}$ and $m_{\tau}$ can take place. For illustrative purpose we have listed the couplings in Eqs. (25)-(27) in terms of $S U(5)$ states. However, at the weak scale, the couplings and the masses of different $S U(3)_{c} \times S U(2)_{L} \times U(1)_{Y}$ representations will differ. This can give a desirable mismatch not only between $m_{b}$ and $m_{\tau}$, but also the experimentally observed values of $m_{\mu} / m_{s}$ and $m_{e} / m_{d}$ if these masses are also generated radiatively. Together with this it would be interesting to study in more details the properties and phenomenology of such grand unified model. However, this is beyond the scope of this paper.

\section{Conclusions}

In this work, we proposed a new extension of MSSM -the FMSSM - with vector like matter at TeV scale, allowing radiative mass generation for down type quarks and charged leptons, thus providing a plausible explanation of why the bottom quark and the tau lepton are so much lighter than the top quark. The $R$-symmetry which we have used provides natural realization of the FMSSM. In particular all couplings are controlled by this symmetry with the $\mu$ and $B_{\mu}$ terms naturally near the $\mathrm{TeV}$ scale. At the same time, the matter parity is automatic and therefore no unwanted baryon and lepton number violating couplings are allowed. Also, the LSP is stable, providing the desired dark matter candidate.

This scenario opens up possibility for having fermiophobic and gaugephobic heavy Higgs sector consisting of the charged $\left(H^{ \pm}\right)$and neutral heavy Higgses $(H$ and $A$ ). We have discussed the phenomenological implications of the model, which are distinct from the usual MSSM, as well as the possible cosmological implications for the cold dark matter. Many of these predictions can be tested by upcoming experiments at the LHC.

\section{Acknowledgement}

We are very grateful to D. Zeppenfeld for many helpful discussions. SN would like to thank the warm hospitality and support of KITP, Santa Barbara, and the organizers of the Workshop "Physics of the Large Hadron Collider" during his participation there when this work was completed. This work was supported in part by the US Department of Energy, Grant Numbers DE-FG02-04ER41306 and DE-FG02-ER46140. This research was also supported in part by the National Science Foundation under Grant Number PHY05-51164. 


\section{References}

[1] J. C. Pati and A. Salam, Phys. Rev. D 10 (1974) 275;

H. Georgi and S. L. Glashow, Phys. Rev. Lett. 32 (1974) 438.

[2] A. G. Akeroyd, Phys. Lett. B 368 (1996) 89;

L. Brucher and R. Santos, Eur. Phys. J. C 12 (2000) 87.

[3] A. E. Nelson and N. Seiberg, Nucl. Phys. B 416 (1994) 46 [arXiv:hep-ph/9309299].

[4] G. F. Giudice and A. Masiero, Phys. Lett. B 206 (1988) 480.

[5] A. H. Chamseddine, R. Arnowitt and P. Nath, Phys. Rev. Lett. 49 (1982) 970;

R. Barbieri, S. Ferrara and C. A. Savoy, Phys. Lett. B 119 (1982) 343.

[6] R. Hempfling, Phys. Rev. D 49 (1994) 6168.

[7] L. J. Hall, R. Rattazzi and U. Sarid, Phys. Rev. D 50 (1994) 7048.

[8] T. Han, R. Mahbubani, D. G. E. Walker and L. T. E. Wang, arXiv:0803.3820 [hep-ph].

[9] M. S. Carena, S. Mrenna and C. E. M. Wagner, Phys. Rev. D 60 (1999) 075010.

[10] K. S. Babu and C. F. Kolda, Phys. Rev. Lett. 84 (2000) 228;

V. M. Abazov et al.(D0 Collaboration), Phys. Rev. D76:092001,2007;

T. Aaltonen et al.(CDF Collaboration), e-Print arXiv:0712.1708[hep-ph];

B.Aubert et al.,(BaBar Collaboration), Phys. Rev. D77:032007,2008.

[11] A. Djouadi, W. Kilian, M. Mihlleitner and P. M. Zerwas, Eur. Phys. J. C. 10,45(1999);

A. Abbasadabi, W. W. Repko, D. A. Dicus, and R. Vega, Phys. Lett. B213,386(1988).

[12] H. Baer, T. Krupovnickas, A. Mustafayev, E. K. Park, S. Profumo and X. Tata, JHEP 0512 (2005) 011.

[13] J. R. Ellis, T. Falk, G. Ganis, K. A. Olive and M. Srednicki, Phys. Lett. B 510 (2001) 236;

J. R. Ellis, K. A. Olive and Y. Santoso, Astropart. Phys. 18 (2003) 395.

[14] P. Pouliot and N. Seiberg, Phys. Lett. B 318 (1993) 169;

R. Barbieri, G. R. Dvali and L. J. Hall, Phys. Lett. B 377 (1996) 76;

K. S. Babu and S. M. Barr, Phys. Lett. B 387 (1996) 87.

[15] B. Stech and Z. Tavartkiladze, Phys. Rev. D 77 (2008) 076009 [arXiv:0802.0894]. 